\title{
Zur Klinik pseudoskleroseähnlicher Krankheitstypen ${ }^{1}$.
}

\author{
Von \\ Josef Gerstmann und Paul Schilder.
}

(Eingegangen am 25. November 1919.)

Die Kenntnis der nicht durch Pyramidenbahnläsion bedingten Bewegungsstörungen hat zwar durch die Arbeiten der letzten Jahre (Anton, Bonhoeffer, Wilson, C. Vogt u. a.) beträchtliche Förderung erfahren, doch sind weder die klinischen noch die pathologisch-anatomischen Fragestellungen hinreichend geklärt. $\mathrm{Zu}$ den bekannten Typen von Erkrankungen mit vorwiegend extrapyramidaler Symptomatologie (Chorea, Paralysis agitans) sind neue progrediente Krankheitsformen hinzugetreten. Es scheint sich um einen geradezu unübersehbaren klinischen Formenreichtum zu handeln und je eifriger man beobachtet, desto mehr eigenartige und schwer deutbare Zustandsbilder treten vor Augen. Ein Teil dieser Fälle hat dadurch besondere Aufmerksamkeit auf sich gezogen, daß mit einer doppelseitigen Striatumerkrankung Leberveränderungen vergesellschaftet waren (Homen, Anton, Wilson). Eine andere Gruppe zeigt jedoch derartige Leberveränderungen nicht. Eigenartige Spannungzustände, die sehr mannigfaltig abgestuft sind, Koordinationsstörungen, Kleinhirnsymptome, choreatisch-athetotische Bewegungen, Tremoren beherrschen das Bild, wobei hervorzuheben ist, daß jede dieser Symptomenbezeichnungen nur ein Sammelname für klinisch und vielleicht auch pathologisch-physiologisch verschiedene Symptome ist. Bei diesem Stand der Dinge ist es nicht nur gerechtfertigt, sondern sogar erforderlich, einschlägige Fälle genau zu analysieren. Der späteren Forschung muß es vorbehalten bleiben, dieses Material ätiologisch und nosologisch zu sichten, die einzelnen Symptome definitiv zu lokalisieren. Diese Gruppe ohne manifeste Leberveränderungen zeigt einerseits Beziehungen zur Pseudosklerose und den verwandten Störungen ${ }^{2}$ ), aber auch zur Chorea Huntington, zur Paralysis agitans und - eine Beziehung, auf die aufmerksam gemacht zu haben das Verdienst Sta uffenbergs ist - zur Atrophia olivo-ponto-cere-

1) Nach einer Dęmonstration im Verein für Psychiatrje und Neurologie in Wien in der Sitzung vom 28. Oktober 1919.

2) Vgl. hierzu die Arbeit yon Economo und Schilder, Eine der Pseudosklerose nahestehende Erkrankung im Praesenium. Erscheint in dieser Zeitschr. 55. 1920 . 
bellaris. Diese Fälle sind in bezug auf die Deutung der Ätiologie und Pathogenese in noch verstärkterem Maße der Unsicherheit ausgesetzt als die genannten Erkrankungen. Es ist ja durchaus nicht geklärt, in welchem Maße exogene und endogene Faktoren an der Auslösung dieser Erkrankungen beteiligt sind. Pathologisch-physiologisch ist allerdings so viel gesichert, daß bei allen diesen Erkrankungen das System Corpus striatum - Nucleus ruber - Cerebellum in seiner Funktion schwer gestört ist. Wir werden im folgenden für dasselbe im Einklang mit Sta uffenberg den Ausdruck extrapyramidales motorisches System gebrauchen. Natürlich bezeichnen die genannten Stationen nur die Hauptpunkte dieses Systems, das ja Teile der Brücke, des Stirnhirns und Temporalhirns gleichfalls umfaßt. Auch ist ja der Ausdruck motorisches System nur ein Verlegenheitsausdruck für sehr mannigfaltige Funktionen, die für die einwandfreie Durchführung von Bewegungsakten nötig sind (Kleinhirn!), die aber nicht ,,motorisch" im engeren Sinne sind. Nach dem Wortsinn wäre ja auch die Apraxie eine extrapyramidale motorische Störung. Wir möchten allerdings diese Störung unter die Bezeichnung nicht einbezogen wissen, trotzdem sehr enge sachliche Beziehungen der Apraxie zu den Störungen des uns interessierenden Apparates bestehen (vgl. hierzu Kleist).

Die Krankheitsgeschichte des Falles verhält sich folgendermaßen:

Veitschegger Ernst, 30 Jahre alt, Werkarbeiter, ledig, wurde am 29. VII. 1919 in die Wiener psychiatriscne Klinik aufgenommen.

Die mit der Schwester des Kranken erhobene Anamnese ergab: Keine hereditäre Belastung. Keine Konsanguinität der Eltern. Als ganz kleines Kind soll Pat. eine Gehirnerkrankung gehabt haben. Sei dann bis 1910 gesund gewesen. Während seiner aktiven Militärzeit sei er auf die Beobachtungsstation in Graz gekommen und soll dort $1 / 2$ Jahr geblieben sein. Sei dann aus dem Heeresverband entlassen worden. Seither könne er schwer stehen resp. gehen, sei schwach im Kopfe. Zeitweilig sei er doch bis zu einem gewissen Grade arbeitsfähig gewesen. Das Leiden habe im Laufe der Zeit immer mehr an Stärke zugenommen. Seit 1914 könne er sich von selbst kaum aufrecht erhalten, bekomme öfters beim Stehen und Gehen Schüttelkrämpfe, falle dabei häufig zusammen. Habe sich bei einer solchen Gelegenheit einmal verbrannt, ein anderes Mal sei er ins Wasser gefallen. Niemals Bewußtseinsstörung. In letzter Zeit zunehmende Verblödung.

Objektiver Befund: Hoher Grad von Demenz. Stark erschwertes und verlangsamtes Auffassungsvermögen. Antwortet erst nach relativ langer Reaktionszeit, nur auf einfache Fragen richtig, kompliziertere Fragen werden sehr mangelhaft aufgefaßt und dementsprechend unvollkommen und unzutreffend beantwortet. Macht schon bei einfachen Rechenaufgaben Fehler. Öfters unmotiviertes, läppisches Lächeln.

Liegt in steifer Körperhaltung stundenlang regungslos im Bett. Führt auf Anrede mit den Händen allerlei Verlegenheitsbewegungen aus, die hin und wieder von eigenartigen Schüttel- resp. Ruckbewegungen im Bereiche der Arme begleitet werden. Aufgetragene Bewegungen werden vom Pat. langsam und träge ausgeführt, wie wenn er zunächst einen gewissen Widerstand zu überwinden hätte.

Sprache langsam, schwerfällig, monoton, etwas undeutlich, zum Teil mangelhaft artikuliert, von pseudobulbärem Typus. Ausdruckslose, starre Gesichtszüge. 
Spontanes Aufstehen (wegen der Spannungszustände) stark erschwert. Beim Aufrichten des Pat. bzw. beim Stehen kommt es zu jähen, groben Zuck- und Ruckbewegungen des Rumpfes gegen die Oberschenkel. Auch in den großen Armgelenken kommt es dabei zu groben, sehr raschen Zuck- und Ruckbewegungen. Noch ausgeprägter sind diese sowie die betreffenden Bewegungsstörungen des Rumpfes beim Gehen. Es ist ein Hin- und Herpendeln des gesamten Oberkörpers, das zu den schwersten Balancestörungen führt. Auffallend ist dabei, daß der Kranke eigentlich gar keine Versuche macht, diese Balancestörungen auszugleichen, das gestörte Körpergleichgewicht wiederzugewinnen. Es scheinen ihm die entsprechenden Antriebe gänzlich zu fehlen, wie er überhaupt einen ausgesprochenen Mangel an Bewegungsantrieben zeigt.

Der Gang selbst ist steif, das Gehen erfordert ausgiebige Unterstützung, er tritt wohl mit der Sohle auf, bleibt aber vor Wechsel des Schrittes mit den Zehenballen am Boden kleben, erstarrt gewissermaßen für einige Augenblicke in dieser Haltung; die Abwicklung der Ballen erfolgt dann glatt. Er zeigt beim Gehen häufig cine ausgesprochene Tendenz nach hinten zu fallen. Das sonst reflektorisch auftretende Suchen der breiteren Basis fällt hier wie beim Stehen mit geschlossenen Augen weg, und er fällt wie ein Klotz steif nach hinten. Der Drehpunkt liegt in den Fersen. Weist bein Gehen die gleichen Bewegungsstörungen am Rumpfe auf wie beim Stehen.

Niedersetzen auf einen Sessel erfolgt steif, ruckartig, so daß er fast hemmungslos auf den Sessel niedersaust. Bei der Aufforderung aufzustehen, erhebt er sich vollständig steif, ruckweise, fällt dabei immer wieder zurück, es scheint, daß er die Kontraktion der Beckenmuskulatur und deren Entspannung weder recht. zeitig noch genügend ausgiebig leisten kann.

Ruhetonus in den oberen Extremitäten nur mäßig, im Bereiche der Rumpfmuskulatur und der unteren Extremitäten in einem sehr beträchtlichen Grade gesteigert. Passiv erteilte Stellungen werden an Armen und Beinen kataleptiform festgehalten, an den ersteren weniger stark ausgesprochen als an den letzteren. Beugt man den Oberkörper beim Sitzen zurück, so gehen die unteren Gliedmaßen steif, durch den Hypertonus fixiert, mit. Passive Bewegungen stoßen an den oberen Extremitäten auf einen sehr geringen, an den unteren Extremitäten auf einen hochgradigen Widerstand. Letzterer ist im allgemeinen ein wächserner, gleichmäßig die Agonisten wie die Antagonisten betreffender, geht nur zeitweise in Treppen, wechselt etwas in der Intensität, steigert sich zwar einigermaßen bei brüsken Bewegungen, nimmt jedoch nie einen federnden Charakter an. Beim Versuch das Bein gegen einen Widerstand von der Unterlage aufzuheben, und bei plötzlichem Nachlassen dieses Widerstandes, geht das Bein nur wenig in die Höhe und wird in dieser Haltung noch über der Unterlage fixiert. Wiederholte passive Bewegungen wirken eher spannungslösend. Noch mohr wirken in diesem Sinne aktive Bewegungen.

Die hier vorliegenden Muskelspannungen sind wohl zum Teil reflektorischer, zum wesentlicheren und erheblicheren Teil jedoch extrapyramidaler Art.

Bemerkt sei noch, daß die Muskelspannungen auch im Schlaf, wenngleich in deutlich vermindertem Grade, nachweisbar sind.

Keine Zeichen von Parese. Aktive Bewegungen in entsprechendem Ausmaße ausführbar, jedoch ausgesprochen langsam, speziell an den unteren Extremitäten durch die Spannungszustände und durch dic Verlangsamung und Erschwerung der Entspannung behindert, steif. Kräftig entwickelte Muskulatur. Keine Adiadochokinese.

Ausgesprochene Verarmung an spontanen Bewegungsantrieben.

Beim Finger-Fingerversuch gegen das Ende der Bewegung zu intentionstremorartiges Überschleudern und Zurückfahren. 
Bei Zielbewegungen (Ergreifen eines Gegenstandes $\mathbf{u}$. dgl.) geht die Bewegung des betreffenden Armes am Anfang relativ gut vonstatten, vor dem Erreichen des Zieles aber setzt ein Zwischenimpuls ein, welcher zumeist in den großen Gelenken (Schultergelenken) angreift und die Hand in einen eigenartigen Tremor versetzt. Dieser eigentümliche Tremor, der nicht nur am Ende der Intention, sondern sehr häufig auch dann sich zeigt, wenn eine erreichte Stellung fixiert werden soll, besteht in regellosen, wild ausfahrenden Ruckbewegungen nach verschiedenen Richtungen, mit einem in der Exkursionsweite wechselnden Hin- und Herpendeln, wobei die Hand zeitweise förmlich im Kreise sich bewegt. Dieses Ausfahren befindet sich in keiner bestimmten Beziehung zur ursprünglichen Bewegungsrichtung, nur ausnahmsweise bleiben die pendelnden Bewegungen in der Richtung der Intention. Ist die Fixation erreicht, dann fallen die Tremorerscheinungen fast völlig aus, insbesondere wenn man minimale Hilfen zur Fixation gibt.

Während der jeweiligen Untersuchungen keine Mitbewegungen zu beobachten.

Keine Störungen der Praxie im engeren Sinne. Zweckhandlungen werden nur durch das beim Abschluß der Bewegung im Bereiche der in Aktion tretenden Extremität sich geltend machende, oben beschriebene eigenartige Ausfahren in entsprechender Weise erschwert und behindert; sie werden aber schließlich intentionsgemä $B$ ausgeführt.

Augenhintergrund obne pathologische Veränderung. Augenbewegungen ungestört, nur langsam. Geruchs- und Geschmacksempfindung ohne Störung. Schluckvermögen intakt. Cornealreflexe lebhaft. Kein Cornealring.

Kein spontaner Nystagmus. Das spontane Zeigen durch die starken ruckartigen Unruhebewegungen gestört, doch gutes Einhalten der Richtung beiderseits. In Romberg-Stellung Schwanken, mit deutlicher Falltendenz nach rückwärts, außerdem die obengenannten, das Körpergleichgewicht stark beeinträchtigenden Ruckbewegungen des Oberkörpers.

Ohrbefund (Dr. Fremel): Trommelfell-Mittelohr-Cochlearapparat beiderseits normal. Linkes Ohr kalt ausgespült: Typischer Nystagmus nach rechts. Typisches Vorbeizeigen beider oberen Extremitäten nach links, bei der zweiten Untersuchung nur von sehr kurzer Dauer und bald von einem richtigen Zeigen gefolgt. Im Romberg-Stellung starke ruckartige Bewegungen des Oberkörpers, dabei Fallen nach rückwärts und bei der ersten Untersuchung auch etwas nachlinks. Keine Beeinflußbarkiet der Fallrichtung durch Änderung der Kopfhaltung. Rechtes Ohr kalt ausgespült: Typischer Nystagmus nach links. Fehlen des Vorbeizeigens nach außen (rechts), im rechten Arm bei der ersten, in beiden Armen bei der zweiten Untersuchung. In Romberg-Stellung Fallen nach rechts bzw. nach rechts-hinten, dabei Fallrichtung durch Veränderung der Kopfstellung im wesentlichen unbeeinflußt.

Lebhafte Bauchdeckenreflexe. Lebhafte Cremaster- und Plantarreflexe. Gesteigerte Patellar- und Achillessehnenreflexe beiderseits. Patellar- und Fußklonus von auffallend gleichmäßigem Charakter. Babinskisches Symptom rechts ganz leicht angedeutet, links positiv.

Linker Fußrücken stark gewölbt, Fuß dadurch kürzer und einem gedrungenen Hohlfuß entsprechend. Zehen links dorsalflektiert. (Der linke Fuß zeigt so eine beträchtliche Ähnlichkeit mit dem sog. ,Friedreich schen Fuß“.)

Die elektrische Prüfung ergab außer einer relativen quantitativen Herabsetzung der direkten faradischen Muskelerregbarkeit und einer gewissen Verlangsamung und Trägheit der Zuckungen sonst keine Besonderheiten.

Die Leberfunktionsprüfung ergab einen negativen Befund. Innere Organe ohne pathologische Veränderungen. 
Zeitweiliges und in seiner Intensität stark wechselndes Auftreten von Harnund Stuhlretention, im wesentlichen auf die Demenz, Ungeschicklichkeit und Spannungszustände in der Muskulatur zurückzuführen.

Wasserman $n$ sche Reaktion im Blut und Liquor negativ. Globulinreaktion, Zellgehalt im Liquor der Norm entsprechend.

Was den Verlauf des Leidens während der bereits 5 Monate dauernden Beobachtungszeit betrifft, so war - abgesehen von einigen, von äußeren Verhältnissen unabhängigen, an manchen Tagen mehr, an anderen weniger ausgeprägten Intensitätsschwankungen - eine gewisse sehr langsam und ganz allmähliche $\mathrm{Zu}$ nahme der Erscheinungen unverkennbar. Jedoch war diese Progredienz nur eine quantitative, graduelle, während qualitative Veränderungen in dem Krankheitsbilde nicht zu verzeichnen waren.

Es handelt sich demnach in dem geschilderten Falle um eine chronisch-progrediente, mit Demenz einhergehende Erkrankung. Im Vordergrunde des Krankheitsbildes stehen: 1. Muskelspannungen. Diese sind nur zu einem geringen Teil auf Läsion der Pyramidenbahn zu beziehen, der wesentlichere Anteil ist extrapyramidaler Genese. 2. Ruckartig ausfahrende Zuckungen der Extremitäten vor dem Erreichen des Zieles pendelnden Charakters (Störungen des Bewegungsabschlusses). Bewegungsstörungen von ähnlichem Typus bei Rumpfbewegungen. 3. Eigenartige Störungen der Körperbalance, vorwiegend beim Gehen und beim Sichaufrichten aus liegender Haltung. Ausfälle vestibulärer Reaktionsbewegungen.

Ad 1. Die Spannungen sind gleichmäßig auf Agonisten und Antagonisten verteilt. Sie nehmen bei brüsken Bewegungen etwas zu. Aktive Bewegungen, wiederholte langsame passive Bewegungen wirken gewöhnlich entspannend. Die Spannungen sind auch im Schlafe - bis zu einem gewissen Grade - vorhanden. Paresen bestehen nicht. Bauchdeckenreflexe sind lebhaft. Auffallend gleichmäßiger Fuß- und Patellarklonus. Babinskisches Symptom (hauptsächlich links) positiv.

Mit Rücksicht auf den positiven Ausfall des letztgenannten Symptoms kann nicht daran gezweifelt werden, daß eine Pyramidenbahnläsion vorliegt. Es liegt nahe, die Zunahme der Spannungen bei brüsken Bewegungen hiermit in Zusammenhang zu bringen. Der Fuß- und Patellarklonus ist eigenartig. Er erinnert in mancher Hinsicht an die Kloni der Paralysis agitans, deren Bedeutung nicht klargestellt ist. Als irgendwie hochgradig kann die Pyramidenbahnläsion nicht veranschlagt werden, denn es bestehen keine Paresen. Die Spannungen sind zweifellos durch die Pyramidenbahnläsion nicht restlos erklärt. Denn sie betreffen - wie gesagt - Agonisten und Antagonisten gleichmäßig und fixieren aktiv eingenommene und passiv gegebene Stellnngen auffallend rasch. Sie gemahnen hierin an die paradoxe Kontraktion, wie sie Westphal bei der Pseudosklerose beschrieben und Strümpell (u. a.) in seinem sog. ,amyostatischen Symptomenkomplex" wiedergefunden hat. Es ist auch daran zu erinnern, daß die Spannungen an den oberen Extremi- 
täten gering sind. Sehr ausgesprochen ist bei unserem Kranken die Bewegungsverarmung, der Mangel an spontanen Bewegungsantrieben. Es ist dieses Symptom sicher nicht auf die Demenz zurückzuführen. Kleist u.a. haben es mit extrapyramidalen Spannungszuständen vergesellschaftet gefunden.

Ad 2. Bei Ziel- und Zweckbewegungen der Hand geht die Bewegung zu Beginn prompt und zweckentsprechend vonstatten. Vor dem Erreichen des Zieles erfolgt jedoch ein ruckweises, jähes Ausfahren, welches durch abnorme Kontraktionen im Bereiche der Schultermuskulatur zustande kommt. Diese ausfahrenden Ruckbewegungen sind mit einem starken, in der Exkursionsweite variierenden Hin- und Herpendeln der betreffenden Extremität verbunden, zeigen in ihrer Richtung keine festgelegte Beziehung zur ursprünglichen Bewegungsrichtung, sind regellos, und erfolgen entweder vor dem Erreichen des Zieles oder wenn die erreichte Stellung fixiert werden soll. Die genannte Bewegungsstörung ist nicht abhängig von der Größe des durchmessenen Weges und von dem Kontraktions- und Spannungszustande der Muskulatur. Sie kennzeichnet sich damit als eine Störung, welche an den Bewegungsabschluß als solchen, an die Bewegungsbremsung gebunden ist ${ }^{\mathbf{1}}$ ). Wir legen auf diese Kriterien Gewicht.

Auch der Intentionstremor der multiplen Sklerose ist an den $\mathrm{Be}-$ wegungsabschluß gebunden. Ferner wirkt in einer Reihe von Pseudosklerosefällen der Bewegungsabschluß versärkend auf die Tremorbewegungen. Der Tremor dieser Krankheiten ist jedoch dadurch charakterisiert, daß er doch meist in der Richtung der ursprünglichen Bewegung und weiters in regelmäßigeren Exkursionen abläuft, während in unserem Falle jegliche Beziehung der Richtung der ausfahrenden Pendelbewegung zur ursprünglichen Bewegungsrichtung fehlt und die pathologischen Bewegungen ein ganz unregelmäßiges Verhalten zeigen. Das ruckartige Ausfahren in unserem Falle ist bedingt durch Zwischenimpulse in einem entfernten großen Gelenk, das bei vielen Bewegungen gar nicht beansprucht wird und im wesentlichen nur fixiert sein sollte. Bei den vorher genannten Tremorformen aber spielt sich die Bewegungsstörung in Muskelgruppen ab, welche an der Bewegung in direkter Weise mitwirken.

$\mathrm{Da}$ ß die hin und her pendelnde Hand in unserem Falle häufig kreisförmige und elliptische Linien beschreibt, ist ein Hinweis dafür, daß die „Synergisten" (soweit dieser Ausdruck bei einer derartigen Bewegung überhaupt zulässig ist) gleichfalls an der Aktion beteiligt sind.

1) Schilder hat für diese Störung die Bezeichnung teleokinetisch vorgeschlagen. (Über Störungen der Bewegungsbremsung usw. Diese Zeitschr. 4r, 1919.) 
Eine weitere Eigentümlichkeit des geschilderten Tremors ist darin gelegen, daß das Ausfahren nicht nur vor dem Erreichen des Zieles auftritt, sondern auch wenn das Ziel erreicht ist und nun die Extremität fixiert werden soll. Ähnliches haben wir auch in gewissen Fällen von Intentionstremor gesehen. Es scheint also, daß die Bewegungsbremsung in zwei Abschnitte zerfällt, nämlich in Bremsung und Fixierung.

Die bisher vorliegenden Untersuchungen machen es wahrscheinlich, daß die Bewegungsbremsung (Teleokinese) an den Kleinhirnapparat geknüpft ist. Babinski hat bei Kleinhirnläsionen Ausbleiben der Bremsung - Hypermetrie, Söderbergh und Schilder ${ }^{1}$ ) vorzeitige Bewegungsbremsung - Bradyteleokinese nachgewiesen. Die engen Beziehungen des Intentionstremors zum Kleinhirn und die Ähnlichkeit des bei Kleinhirnläsionen beobachteten Tremors mit dem Intentionstremor sind bekannt (vgl. insbesondere die einschlägigen Arbeiten Babinskis). Es liegt nach allem nahe, auch den eigenartigen, in dieser Form bisher nicht beschriebenen Tremor unseres Falles, da er an die Bewegungsbremsung gebunden ist, auf eine Läsion des Kleinhirnapparates zu beziehen.

Diese Ermittlungen über den Tremor der Extremitäten verhelfen uns auch zu einem Verständnis der Bewegungsstörungen im Bereiche des Rumpfes beim Stehen und Gehen. Beim Gehen und Stehen handelt es sich ja um fortwährende Regulierungen von Zielbewegungen und um immer neu einsetzende Sicherungen neu gewonnener Haltungen. Eine ähnlich exakte Analyse, wie wir sie soeben für die Bewegungsstörungen der Extremitäten geliefert haben, ist freilich bei den Rumpfbewegungen nicht möglich. Doch ergibt die Beobachtung auch hier pendelnde und ausfahrende Ruckbewegungen in den großen Gelenken, die Rumpf und Beine verbinden. Sehr häufig treten dieselben jedoch auch in den Schultergelenken und in den Gelenken auf, in welchen der Hals gegen den Rumpf bewegt wird. Daß die Störung beim Stehen so ausgesprochen ist, dürfte wohl damit in Zusammenhang zu bringen sein, daß auch die Haltungsfixation, wie oben erwähnt, mit dem Tremor vergesellschaftet ist.

Ad 3. Ein Teil der schweren Störungen der Balance, die der Patient bietet, dürfte durch die beschriebenen Schleuderbewegungen des Rumpfes erklärt sein. Es ist jedoch zu beachten, daß auch hiervon abgesehen der Patient die Tendenz zeigt, jäh nach hinten zu fallen. Hierbei ist auffallend, daß er keine Versuche unternimmt, das Gleichgewicht wiederzugewinnen. Er kippt geradezu um die Ferse als Drehpunkt um. Dieses Verhalten muß den Verdacht einer Wurmaffektion wachrufen. Für eine solche ist ja wesentlich, daß die für die Körperbalance nötigen

1) Schilder hat noch vor der Mahnung Söderberghs (Neurol. Centralbl. Juni 1919) dessen Arbeiten zitiert. 
Innervationen nicht zueinander abgestimmt werden (Asynergie cérébelleuse). Auch bei unserem Kranken fehlt diese Abstimmung. Es handelt sich jedoch nicht um das typische Zurückbleiben des Körpers hinter den Beinen, so daß man sich um weitere Stützen dieser Anschauung umsehen muß. Hier greift die Funktionsprüfung des Vestibularapparates ergänzend ein. Jedenfalls sind die Fallreaktionen nicht typisch auslösbar was die Vermutung zu stützen geeignet ist, daß eine Wurmaffektion, vorliege. Allerdings erschwert die spontane Unruhe des Kranken beim Stehen die Prüfung und Beurteilung. Das Fehlen der Zeigereaktion rechts kann wohl nur auf einer Kleinhirnläsion beruhen (rechte Hemisphäre).

Versuchen wir jetzt ein Gesamtbild übsr die Lokalisation des Prozesses zu gewinnen, so muß gesagt werden, daß der bei Bewegungsabschluß auftretende ruckweise auffahrende, eigenartige Tremor unseres Falles, die Rumpfkoordinationsstörungen, der Ausfall an Zeige- und Fallreaktionen eine Läsion des cerebellaren Systems vermuten lassen. Die Störung dürfte vorwiegend im Kleinhirnwurm und den benachbarten Partien lokalisiert sein. Selbstverständlich müßte eine ausgiebige Ausschaltung zugehöriger Bahnen den gleichen Effekt haben. Die gute Diadochokinese an den Extremitäten ist beachtenswert. Trotzdem ist eine Mitschädigung der rechten Kleinhirnhemisphäre durch den Ausfall der Zeigereaktion gesichert.

Zur Erklärung der extrapyramidalen Spannungen muß keine erhebliche Schädigung des Corpus striatum angenommen werden. In einem Falle Stauffenbergs mit ausgeprägten Spannungszuständen war neben einer ausgesprochenen Atrophia olivo-ponto-cerebellaris eine nur geringfügige Striatumschädigung nachweisbar und in einem Falle von Economo und Schilder war bei geschädigtem Kleinhirn eine relativ wenig ausgedehnte Striatumschädigung ausreichend, um schwere Rigoren hervorzurufen. Steifigkeit der Bewegungen hat bereits Kleist als wesentlichen Zug in der Atrophia olivo-ponto-cerebellaris hervorgehoben. Sta uffenbergs umfassende und kritische Zusammenstellung führt das erneut vor Augen.

Die Verarmung an Spontanbewegungen hebt Kleist aus den Schilderungen der Wilsonfälle hervor. Sie ist ein Lokalsymptom, dessen genaue Auswertung noch unbekannt ist (cerebrocerebellare Bahnen?).

Daß die Pyramidenbahn gleichfalls lädiert ist, haben wir bereits betont. Eine Rindenschädigung ist mit Rücksicht auf die hochgradige Demenz anzunehmen.

Es handelt sich demnach jedenfalls um eine diffuse Erkrankung des Gehirns, bei der das Kleinhirnsystem und die mit ihm in funktioneller Verbindung stehenden Apparate besonders betroffen sind.

Die klinische Deutung hat zunächst die multiple Sklerose auszu- 
schließen. Gegen diese spricht: der chronisch-progrediente Verlauf ohne Remissionen, das Fehlen von Augensymptomen (kein Nystagmus, kein Doppelsehen, keine Zeichen von Opticusatrophie), das Vorhandensein von lebhaften Bauchdeckenreflexen, die Geringfügigkeit von Erscheinungen seitens der Pyramidenbahnen.

Eine Lues cerebri kommt nach dem negativen Ausfall der vier Reaktionen sowie nach dem ganzen Verlauf nicht in Frage.

Die Anamnese, speziell das Fehlen einer hereditären Belastung und Konsanguinität, spricht gegen die ataktische Heredodegeneration, ebenso der ganze Symptomenkomplex. Es läßt sich das hier vorliegende Krankheitsbild nach unserer bisherigen Erfahrungen weder mit der spinalen Form derselben, der Friedreichschen Ataxie (wegen Lebhaftigkeit der Sehnenreflexe, Mangel einer charakteristischen Sprachstörung, wegen der oben beschriebenen Spannungszustände $\mathfrak{u}$. dgl.), noch mit dem Mdrieschen Typus, der sog. Hérédoataxie cérébelleuse in Einklang bringen. Zeichen konstitutioneller Minderwertigkeit finden sich nicht, wenn man nicht etwa die dem sog. Fried reich schen Fuß entsprechende Deformität des linken Fußes bei unserem Kranken als solches ansehen will. Doch führen einzelne Autoren diese Anomalie auf Muskelspannungen zurück. Trotzdem wir das Kleinhirn in das Zentrum unserer Betrachtung gerückt haben, fällt es uns schwer, den Fall einer der bekannten Kleinhirnerkrankungen anzuglièdern. Allerdings ist ja die cerebellare Heredoataxie ein noch ungeklärtes Krankheitsbild, einzelne der bei ihr differentialdiagnostisch verwerteten Momente können in manchen Fällen der Erkrankung fehlen, so daß von der letzteren Brücken führen zu einer Affektion, mit der unser Fall größere Ähnlichkeiten bietet, nämlich zu dem bereits erwähnten Krankheitsbilde der Atrophia olivo-ponto-cerebellaris. Insbesondere ein Fall Ficklers, in dem neben der Cerebellaratrophie eine hochgradige Atrophie des Stirnhirns und eine weitgehende Degeneration der Ganglienzellen des Linsenkernes sich fanden, zeigt klinisch beträchtliche Ähnlichkeiten zu unserem Fall. Nur sind in jenem Fall die Spannungen von anderem Typus, treten lediglich bei Bewegungen auf, die Sprache ist nicht pseudobulbär (wie in unserem Falle), sondern explosiv, die Inkoordination tritt stärker hervor. Von den Fällen der ebengenannten Erkrankung ist unser Fall durch sein relativ frühzeitiges Einsetzen geschieden. In der Zusammenstellung Sta uffenbergs ${ }^{1}$ ) beginnen nur der Fall Menzels und der eine Fall von Thomas (6) in ähnlich frühem Alter. Dieser erinnert allerdings sehr an die cerebellare Heredoataxie. Man kann sich des Eindruckes nicht erwehren, daß die Stauffenbergsche

1) Stauffenberg, Zur Kenntnis des extrapyramidalen motorischen Systems und Mitteilung eines Falles von sog. "Atrophie olivo-ponto-cérébelleuse". Diese Zeitschr. 39. 1919. 
Gruppe selbst inhomogen ist und daß selbst mit der Zuteilung zu ihr nichts Wesentliches gewonnen wäre.

Die meisten Deckungsstellen zeigt das klinische Bild unseres Falles mit der Pseudosklerose. Die Rigoren, die tremorartigen Ruckbewegungen, die einzelnen Zeichen von Pyramidenläsion, die Demenz passen sehr gut zu dem Bilde dieser Erkrankung, für die auch der Verlauf spräche. Die Koordinationsstörung wäre allerdings ungewöhnlich. Noch mehr spricht gegen Pseudosklerose, daß die Untersuchung der Leber (Palpation, Funktionsprüfung) keine pathologischen Veränderungen ergab.

Alter, Koordinationsstörung, die geschilderten Störungen der Bewegungsbremsung usw. sprechen mit Entschiedenheit gegen die Paralysis agitans.

So zeigt es sich, daß der Fall sich keiner der bekannten Erkrankungsformen zwanglos angliedern läßt. Er vermehrt somit den Formenreichtum jener chronisch-progredienten Erkrankungstypen, in denen die Schädigung des extrapyramidalen motorischen Systems in dem eingangs genannten Sinne das klinische Bild beherrscht. 